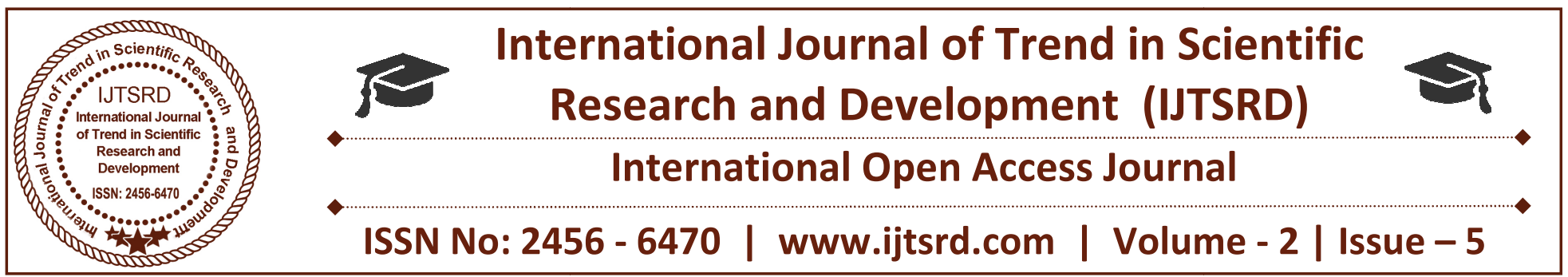

\title{
Literature Review on Single Image Super Resolution
}

\author{
Shalini Dubey ${ }^{1}$, Prof. Pankaj Sahu ${ }^{2}$, Prof. Surya Bazal ${ }^{2}$ \\ ${ }^{1}$ Research Scholar, ${ }^{2}$ Assistant Professor \\ Department of ECE, GGITS, Jabalpur, Madhya Pradesh, India
}

\begin{abstract}
In this paper, a detailed survey study on single image super-resolution (SR) has been presented, which aims at recovering a high-resolution (HR) image from a given low-resolution (LR) one. It is always the research emphasis because of the requirement of higher definition video displaying, such as the new generation of Ultra High Definition (UHD) TVs. Super-resolution (SR) is a popular topic of image processing that focuses on the enhancement of image resolution. In general, SR takes one or several lowresolution (LR) images as input and maps them as output images with high resolution (HR), which has been widely applied in remote sensing, medical imaging, biometric identification.
\end{abstract}

Keyword: Super Resolution, Image Reconstruction, Single Image Resolution Techniques, Resolution Enhancement.

\section{INTRODUCTION}

Image super resolution is a image processing algorithms that produce high quality, high resolution (HR) images from a set of low quality, low resolution (LR) images or from a single image. The SR image reconstruction is useful in many practical cases where multiple frames of the same scene can be obtained, including medical imaging, satellite imaging, and video applications [17]. The basic premise for increasing the spatial resolution in SR techniques is the availability of multiple LR images captured from the same scene. The set of source low resolution (LR) images captures only a finite amount of information from a scene; the goal of SR is to extract the independent information from each image in that set and combine the information into a single high resolution (HR) image. The requirement is of $\mathrm{SR}$ is that each LR image must contain some information that is unique to that image [3]. The super resolution method is to take more samples of the scene so as to get some extra information which can be used, while merging the samples to get a high resolution image. These samples can be acquired by sub-pixel shifts, by changing scene, by changing the amount of blur [14] .HR means that pixel density within an image is high, and therefore an HR image can offer more details that are important in many applications, The major advantage of the super resolution approach is that it may cost less and the existing LR imaging systems can be still utilized. Synthetic zooming of region of interest (ROI) is another important application in surveillance, forensic, scientific, medical, and satellite imaging [13]. This application is most suitable for magnifying objects in the scene such as the face of a criminal or the license plate of a car [16].

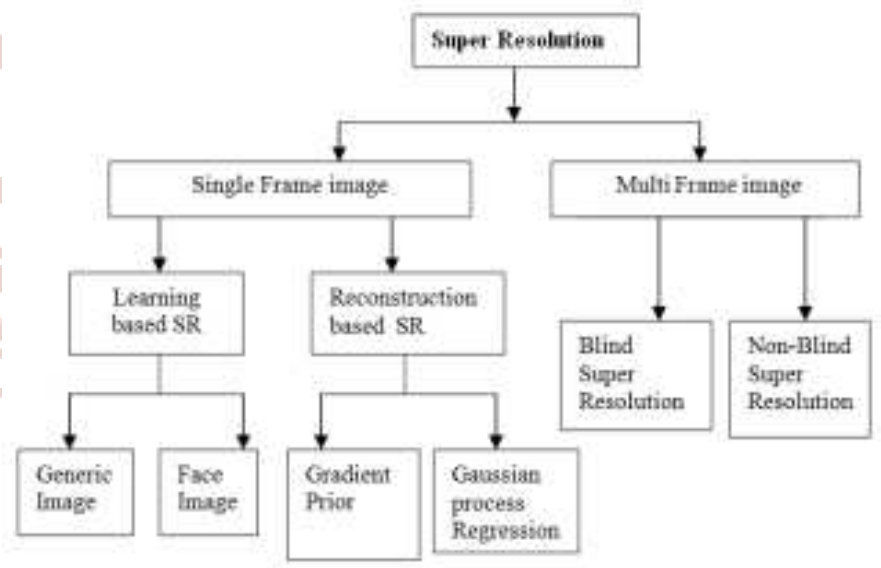

Figure 1: Classification of Super Resolution Techniques

\section{LITERATURE SURVEY}

[1] This study proposes a novel super-resolution regularization model based on adaptive sparse representation and self-learning frameworks. The fidelity term in the model ensures that the 
reconstructed image is consistent with the observation image. The adaptive sparsity regularization term constrains the reconstructed image with an adaptive sparse representation, which successfully harmonizes the sparse representation and the collaborative representation adaptively via producing suitable coefficients. To construct a more effective dictionary, the high-frequency features from the underlying image patches are extracted, and the dictionary learning and sparse representation are integrated. To this end, the alternating minimization algorithm is used to divide this model into three sub-problems, and the alternating direction method of multipliers and iterative back-projection method are used to solve the sub-problems. To illustrate the effectiveness of the proposed method, additional experiments are conducted on some generic images. Compared with some state-of-the-art algorithms, the experimental results demonstrate that the proposed method achieves better results in terms of both visual quality and noise immunity.

[2] Traditional works have shown that patches in a natural image tend to redundantly recur many times inside the image, both within the same scale, as well as across different scales. Make full use of these multi-scale information can improve the image restoration performance. However, the current proposed deep learning based restoration methods do not take the multi-scale information into account. In this paper, we propose a dilated convolution based inception module to learn multi-scale information and design a deep network for single image superresolution. Different dilated convolution learns different scale feature, then the inception module concatenates all these features to fuse multi-scale information. In order to increase the reception field of our network to catch more contextual information, we cascade multiple inception modules to constitute a deep network to conduct single image superresolution.

[3] Y. Sun Et-al, presented a compressive sensing based on a redundant dictionary has been successfully applied in super resolution imaging. However, due to the neglect of the local and nonlocal interactions of patches of a single image, the reconstructed results are not satisfactory in noise suppression and edge sharpness. In this paper, we propose an improved method by adding steering kernel regression and a nonlocal means filter as two regularization terms and use an efficient clustering sub-dictionary learning scheme. We further demonstrate better results on true images in terms of traditional image quality assessment metrics.

[4]Y. Sun Et-al, presented an observation for medical imaging and astronomical, high-resolution (HR) images are urgently desired and required. In recent years, many researchers have proposed various ways to achieve the goal of image super-resolution (SR), ranging from simple linear interpolation schemes to nonlinear complex methods. In this paper, we deal with the SR reconstruction problem based on the theory of compressive sensing, which uses a redundant dictionary instead of a conventional orthogonal basis. We further demonstrate better results on true images in terms of peak signal-to-noise ratio (PSNR) and root mean-square error (RMSE) and give several important improvements, compared with other methods.

[5] M. Agrawal et-al, presented a technique for enhancing resolution of images by interpolating high frequency sub-bands generated using lifting wavelet transform (LWT) and spatial information of input low resolution (LR) image. Stationary wavelet transform (SWT) is used at intermediate stage for edge enhancement. The input image is decomposed using LWT in order to generate high frequency (HF) subbands. The generated HF sub bands are interpolated further. Different high frequency sub-bands obtained through SWT are added to correct the estimated HF sub bands. The input LR image is interpolated in parallel. All these sub-bands and estimated LR image are reconstructed by inverse lifting wavelet transformation (ILWT) to produce high resolution image. The qualitative, quantitative and visual images of the described technique show the superiority of the proposed method over conventional and state-of-theart methods.

[6] H. Ashikaga et-al, presented Single-image super resolution is a process of obtaining a high-resolution image from a set of low-resolution observations by signal processing. While super resolution has been demonstrated to improve image quality in scaled down images in the image domain, its effects on the Fourier-based image acquisition technique, such as MRI, remains unknown. We performed highresolution ex vivo late gadolinium enhancement (LGE) magnetic resonance imaging $(0.4 \times 0.4 \times 0.4$ $\mathrm{mm} 3$ ) in position fraction swine hearts (n D 24). The swine hearts were divided into the training set ( $n$ D 
14) and the test set (n D 10), and in all hearts, lowresolution images were simulated from the highresolution images. In the training set, super resolution dictionaries with pairs of small matching patches of the high- and low-resolution images were created. In the test set, super resolution recovered high-resolution images from low-resolution images using the dictionaries. The same algorithm was also applied to patient LGE (n D 4) to assess its effects. Compared with interpolated images, super resolution significantly improved basic image quality indices ( $\mathrm{P}$ $<0.001)$. Super resolution using Fourier-based zero padding achieved the best image quality. However, the magnitude of improvement was small in images with zero padding. Super resolution substantially improved the spatial resolution of the patient LGE images by sharpening the edges of the heart and the scar.

[7] E. Faramarzi et-al, presented , a unified blind method for multi-image super-resolution (MISR or SR), single-image blur deconvolution (SIBD), and multi-image blur deconvolution (MIBD) of lowresolution (LR) images degraded by linear spaceinvariant (LSI) blur, aliasing, and additive white Gaussian noise (AWGN). The proposed approach is based on alternating minimization (AM) of a new cost function with respect to the unknown high-resolution (HR) image and blurs. The regularization term for the HR image is based upon the Huber-Markov random field (HMRF) model, which is a type of variational integral that exploits the piecewise smooth nature of the HR image. The blur estimation process is supported by an edge-emphasizing smoothing operation, which improves the quality of blur estimates by enhancing strong soft edges toward step edges, while filtering out weak structures. The parameters are updated gradually so that the number of salient edges used for blur estimation increases at each iteration. For better performance, the blur estimation is done in the filter domain rather than the pixel domain, i.e., using the gradients of the LR and HR images. The regularization term for the blur is Gaussian (L2 norm), which allows for fast non iterative optimization in the frequency domain. We accelerate the processing time of SR reconstruction by separating the up sampling and registration processes from the optimization procedure. Simulation results on both synthetic and real-life images (from a novel computational imager) confirm the robustness and effectiveness of the proposed method.

\section{VARIOUS EXISTING \\ RESOLUTION TECHNIQUES \\ SUPER-}

The generation of the low resolution image can be modeled as a combination of smoothing and downsampling operation of natural scenes by low quality sensors. Super resolution is the inverse problem of this generation process. One criteria of solving this inverse problem is minimizing the reconstruction error. Various methods are proposed in literature to deal with the inverse problem. In following section I categorize the different SR methods available in existing paper.

\section{A. Interpolation Methods}

Image interpolation is the process of converting the image from one resolution to other resolution. This process is performed on a one dimension basis row by row and then column by column. Image interpolation estimates the intermediate pixel between the known pixels by using different interpolation kernel.

\section{Nearest Neighbor Interpolation}

Nearest neighbor interpolation is the simplest interpolation from the computational point of view. In this, each output interpolated pixel assign the value of nearest sample point in the input image [2]. This process just displaces the intensity from reference to interpolated one so it does not change the histogram. It preserves the sharpness and does not produce the blurring effect but produce aliasing.

\section{Bi-linear Interpolation}

In Bi-linear interpolation the intensity at a point is determined from weighted some of intensity at four pixel closet to it. It changes the intensity so histogram is also change. It slightly smooths the image but does not create an aliasing effect.

\section{Bi-cubic Interpolation}

In cubic interpolation intensity at point is estimated from the intensity of 16 closest to it. The basis function is Bi-cubic gives smooth image but computationally demanding.

\section{$>$ B-spline Interpolation}

Spline interpolation is the form of interpolation where interpolant is a special piecewise polynomial called a spline. There is a whole family of the basis function used in interpolation which is given as [2]. Higher order interpolation is much more used when image required many rotation and distortion in separate step. 
However, for single step enhancement is increased processing time.

\section{> Hybrid Approach of Interpolation}

In 2008, H. Aftab et al. [3] proposed a new hybrid interpolation method in which the interpolation at edges is carried out using the covariance based method and interpolation at smooth area is done by using iterative curvature based method. After finding edges and smooth area using information from the neighborhood pixels edge is interpolated using covariance based method. The covariance coefficient of HR image is obtaining using covariance parameter of LR image. In smooth are a curvature interpolation is carried out by first performing bilinear interpolation along the direction where the second derivative is lower and in diagonal case the difference between diagonal is calculated and use bilinear interpolation where the intensity difference is less. This method has significant advantage in terms of the processing time, peak signal to noise ratio and visual quality compared to bilinear, bi-cubic and nearest neighbor.

\section{B. Iterative back projection algorithm}

In this algorithm [1]-[3] back projection error is used to construct super resolution image. In this approach the HR image is estimated by back projecting the error between the simulated LR image and captured LR image. This process is repeated several times to minimize the cost function and each step estimate the HR image by back-projecting the error. The main advantage of this method is that this method converges rapidly, less complexity and low-less number of iteration is required. In recently numbers of improvements are used with this approach which is different edge preserving mechanisms.

\section{Robust Learning-Based Super-Resolution}

This algorithm [5] synthesizes a high-resolution image based on learning patch pairs of low- and highresolution images. However, since a low resolution patch is usually mapped to multiple high-resolution patches, unwanted artifacts or blurring can appear in super-resolved images. In this paper, a novel approach to generate a high quality, high-resolution image without introducing noticeable artifacts. Introducing robust statistics to a learning-based super resolution, we efficiently reject outliers which cause artifacts. Global and local constraints are also applied to produce a more reliable high- resolution image. Learning-based super-resolution algorithms are generally known to provide HR images of high quality. However, their practical problem is the one- to- multiple mapping of an LR patch to HR patches, which results in image quality degradation.

\section{An Efficient Example-Based Approach for Image Super-Resolution}

This algorithm [6], [7] uses learning method to construct super resolution image. The main contributions of these algorithms are: (1) a class specific predictor is designed for each class in our example-based super-resolution algorithm, this can improve the performance in terms of visual quality and computational cost; and (2) different types of training set are investigated so that a more effective training set can be obtained. The classification is performed based on vector quantization (VQ), and then a simple and accurate predictor for each category, i.e. a class-specific predictor, can be trained easily using the example patch-pairs of that particular category. These class specific predictors are used to estimate, and then to reconstruct, the high-frequency components of a HR image. Hence, having classified a LR patch into one of the categories, the highfrequency content can be predicted without searching a large set of LR-HR patch-pairs.

\section{E. Learning Based Super Resolution using Direction lets}

In this algorithm [9] example based method using direction lets (skewed anisotropic wavelet transform) are used to generate high resolution image. It does scaling and filtering along a selected pair of direction not necessary horizontal and vertical like wavelet transform. In this approach the training set is generated by subdividing HR images and LR images into the patches of size $8 * 8$ and $4 * 4$ respectably. And then best pair of the direction is assign to each pair from five set of directions $[(0,90),(0,45),(0,-45),(90$,$45),(90,45)]$ and then grouping the patches according to direction which reduce the searching time. Input LR image is contrast normalized and then subdivided into $4 * 4$ patches. Each patch is decomposed into eight bands passing using direction lets. The directional coefficient of six bands HL,HH,VL,VH,DL,DH are learn from training set. Minimum absolute difference MAD criterion is used to select the direction lets coefficient. For AL and AH cubic interpolated LR image is used. These learned coefficients are used to obtain SR image by taking inverse direction lets transform. At the end contrast normalize is undo. Simple wavelet which is isotropic and does not follow the edges results in the artifacts which are removed in this case. 


\section{CONCLUSION}

The SR imaging has been one of the fundamental image processing research areas. It can overcome or compensate the inherent hardware limitations of the imaging system to provide a clearer image with a richer and informative content. It can also be served as an appreciable front-end pre-processing stage to facilitate various image processing applications to improve their targeted terminal performance. In this survey paper, our goal is to offer new perspectives and out looks of SR imaging research, besides giving an updated overview of existing SR algorithms. It is our hope that this work could inspire more image processing researchers endeavouring on this fascinating topic and developing more novel SR techniques along the way. Hence, we propose a wavelet based super-resolution technique, which will be of the category of interpolative methods. It will be based on sparse representation property of the wavelets.

\section{REFERENCES}

1. Jianwei Zhao et-al, "Image super-resolution via adaptive sparse representation and self-learning", Published in IEEE IET Computer Vision, Volume: 12, Issue 5, 2018.

2. Wuzhen Shi et-al, "Single Image SuperResolution with Dilated Convolution based MultiScale Information Learning Inception Module", IEEE International Conference on Image Processing (ICIP), 2017.

3. Y. Sun, G. Gu, X. Sui, Y. Liu, and C. Yang, "Compressive Super-resolution Imaging Based on Local and Nonlocal Regularizations," IEEE Photonics Journal, Volume 8, No. 1, 2016.

4. Y. Sun, G. Gu, X. Sui, Y. Liu, and C. Yang, "Single image super-resolution using compressive sensing with a redundant dictionary," IEEE Photonics Journal, volume 7, No. 2, 2015.

5. Mayank Agrawal, Ratnakar Dash, "Image Resolution Enhancement Using Lifting Wavelet and Stationary Wavelet Transform", IEEE International Conference on Electronic Systems, Signal Processing and Computing Technologies (ICESC), 2014.

6. Mayank Agrawal, Ratnakar Dash, "Image Superresolution by Interpolating High Frequency Subbands of Image using Surface Fitting", IEEE International Conference on Signal Processing and Communication (ICSC), 2014.
7. Do Kyung Shin, Young Shik Moon, "SuperResolution Image Reconstruction Using Wavelet Based Patch and Discrete Wavelet Transform", Journal of Signal Processing Systems, Springer, Volume 81, Issue 1, pp 71-81, 2014.

8. V. PrasathR. Buvanesvari, N. Thilartham, K. Nirosha, "Image Super Resolution Reconstruction Using Wavelet Transform Method", International Journal of Innovative Technology and Exploring Engineering, Volume-3, Issue-9, 2014.

9. K. Zhang, X. Gao, D. Tao, and X. Li, "Single image super-resolution with non-local means and steering kernel regression," IEEE Trans. Image Process., vol. 21, no. 11, pp. 4544-4556, Nov. 2012.

10. G. Yu, G. Sapiro, and S. Mallat, "Solving inverse problems with piecewise linear estimators: From Gaussian mixture models to structured sparsity," IEEE Trans. Image Process., vol. 21, no. 5, pp. 2481 -2499, May 2012.

11. W. Dong, L. Zhang, G. Shi, and X. Wu, "Image deblurring and super-resolution by adaptive sparse domain selection and adaptive regularization," IEEE Trans. Image Process., vol. 20, no. 7, pp. 1838-1857, Jul. 2011.

12. L. Zhang, L. Zhang, X. Mou, and D. Zhang, "FSIM: A feature similarity index for image quality assessment," IEEE Trans. Image Process., vol. 20, no. 8, pp. 2378-2386, Aug. 2011.

13. J. Yang, J. Wright, T. S. Huang, and Y. Ma, "Image super-resolution via sparse representation," IEEE Trans. Image Process., vol. 19, no. 11, pp. $2861-2873$, Nov. 2010.

14. X. Zhang, M. Burger, X. Bresson, and S. Osher, "Bregmanized nonlocal regularization for deconvolution and sparse reconstruction," SIAM J. Imag. Sci., vol. 3, no. 3, pp. 253-276, Jul. 2010.

15. H. Zhang, J. Yang, Y. Zhang, and T. S. Huang, "Non-local kernel regression for image and video restoration," in Proc. Eur. Conf. Comput. Vis., 2010, pp. 566-579.

16. J. Mairal, F. Bach, J. Ponce, G. Sapiro, and A. Zisserman, "Non-local sparse models for image restoration," in Proc. IEEE Conf. Comput. Vis. Pattern Recog., 2009, pp. 2272-2279.

17. M. Protter, M. Elad, H. Takeda, and P. Milanfar, "Generalizing the nonlocal-means to super- 
resolution reconstruction," IEEE Trans. Image Process., vol. 18, no. 1, pp. 36-51, Jan. 2009.

18. P. Sen and S. Darabi, "Compressive image superresolution," in Proc. Conf. 43rd Asilomar Conf. Signals, System Computer, pp. 1235-1242, 2009

19. M. Li and T. Nguyen, "Markov random field model-based edge-directed image interpolation," IEEE Trans. Image Process., vol. 17, no. 7, pp. $1121-1128$, Jul. 2008.
20. R. Baraniuk, "Compressive sensing," IEEE Signal Process. Mag., vol. 24, no. 4, pp. 118-121, Jul. 2007.

21. H. Takeda, S. Farsiu, and P. Milanfar, "Kernel regression for image processing and reconstruction," IEEE Trans. Image Process., vol. 16, no. 2, pp. 349-366, Feb. 2007.

22. L. Zhang and $\mathrm{X}$. Wu, "An edge-guided image interpolation algorithm via directional filtering and data fusion," IEEE Trans. Image Process., vol. 15, no. 8, pp. 2226-2238, Aug. 2006.

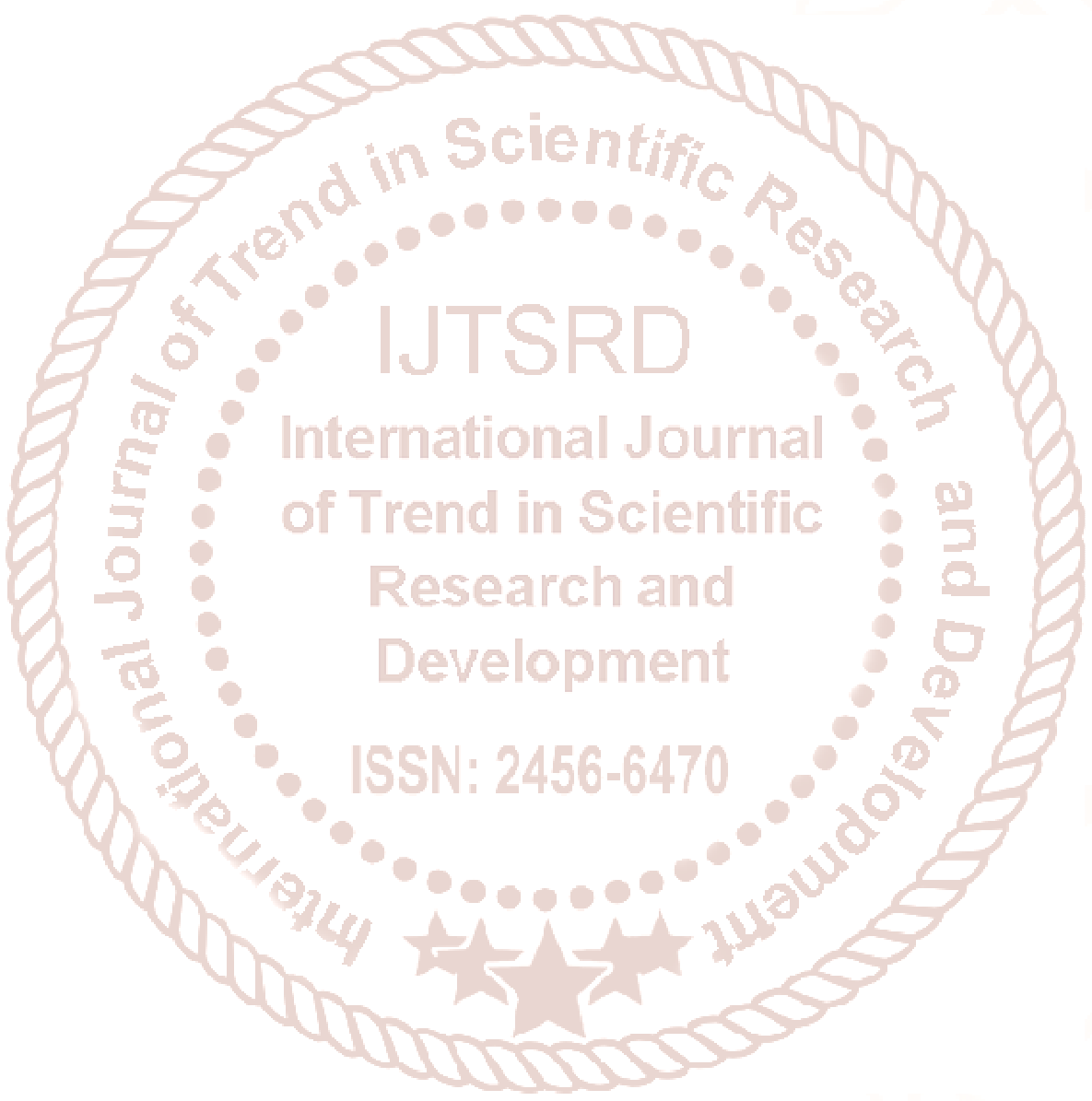

